\title{
Impact of a charged neighboring particle on Förster Resonance Energy Transfer (FRET)
}

\author{
Champi Abeywickrama* and Malin Premaratne ${ }^{\dagger}$ \\ Advanced Computing and Simulation Laboratory $(A \chi L)$, \\ Department of Electrical and Computer Systems Engineering, \\ Monash University, Clayton, Victoria 3800, Australia.
}

Sarath D. Gunapala

Jet Propulsion Laboratory, California Institute of Technology, Pasadena, California 91109, USA.

\author{
David L. Andrews \\ School of Chemistry, University of East Anglia, Norwich Research Park, Norwich NR4 7TJ, United Kingdom
}

(Dated: November 15, 2018)

\begin{abstract}
Förster Resonance Energy Transfer (FRET) is an important physical phenomenon which demands precise control over FRET rate for its wide range of applications. Hence, enhancing the FRET rate by different techniques has been extensively studied in the literature. Research indicates that introducing additional particles to a system consisting of a donor-acceptor pair can change the behaviour of FRET in the system. One such technique is utilizing the collective oscillations of the surface electrons of a neighboring electrically-neutral metal nanoparticle (MNP). However, the perceived changes on FRET rate between donor-acceptor when the MNP carry excess electrical potential is yet unknown. In this paper, we study these changes by introducing a MNP with an electrical potential applied on it, in the proximity of an excited donor and a ground state acceptor pair. We deploy the classical Green's tensor, which behaves as a link between quantum and classical electrodynamics, to express the FRET rate in the system, assuming a spatially persistent local dielectric response for the MNP. We analyze the electrical potential at the acceptor position due to the changed dipole moment of the donor molecule as a result of the electric field induced at the donor position and obtain FRET rate of the system based on these calculations. This model considers capricious locations and orientations of the two molecular dipole moments with regard to the location of the spherical MNP. We present the enhancement of FRET rate, predominantly due to both the surface plasmon excitations and the electrical potential carried by the neighboring MNP. We obtain the results by varying the separation distance between the molecules and the MNP, the transition frequency of the donor-acceptor pair and the size of the metallic sphere. Specifically, we demonstrate that a donor-acceptor pair placed in the vicinity of a electrically-nonneutral Silver MNP exhibits a remarkable improvement in the FRET rate with the amount of potential applied on the MNP. Furthermore, the aggregate FRET enhancement is determined by other characteristics such as location of the donor, transition frequency, separation distances and the radius of the MNP. In essence, these findings reveal an approach to realize enhanced FRET rate in a larger span in a more controlled manner that is desirable in many FRET-based applications including spectroscopic measurements.
\end{abstract}

\section{INTRODUCTION}

The energy transfer between an excited and a groundstate chemical entity is an important phenomenon that is extremely useful for real world applications [1,2]. This energy transfer may occur with or without the emission of a photon [3-5] in three possible forms: Dexter transfer [6], Förster Resonance Energy Transfer (FRET) [7, 8] and radiative transfer [9-11]. Here we focus on FRET, which is a radiationless energy transfer mechanism from a donor to an acceptor molecule. The donor in its excited state transmits energy to a ground state acceptor, owing to the radiationless dipole-dipole interaction known as Coulombic coupling. The donor transition takes place

\footnotetext{
* champi.abeywickrama@monash.edu

$\dagger$ malin.premaratne@monash.edu
}

from a higher energy state to a lower energy state whilst the acceptor transits energy from a lower energy state to a higher energy state. This radiationless process is acknowledged as an exchange of a virtual photon between the donor and the acceptor [12]. This idea was first hypothesized by Förster in 1946 [7] and the ideal dipole approximation is implicit in the Förster equation $[13,14]$.

FRET is found to be nearly $100 \%[15,16]$ efficient in naturally occurring phenomena such as photosynthesis $[15,17]$, where the solar energy captured by the photosynthetic antenna units of plants are transferred to a reaction center by a series of jumps between other chromophores. Due to its promising prospects for a wide range of applications, a plethora of studies have been conducted on FRET to match the light harvesting efficiency of these natural events. These applications spread over artificial light-harvesting devices [1822 ], biomedical sensing [23-25], quantum computation 
[26-29], nano-emitters and spasers [30-34]. Intermolecular and Intramolecular FRET-based biosensors are commonly used in cellular physiology, cellular molecular dynamics, and cell-cell interactions today [35]. However, FRET rate degrades inversely proportional to the sixth power of the interspace between the energy exchanging constituents, restricting its reach to a few nanometers. As a result, FRET based measurements are considered to be much sensitive in the context of molecular biology [36] and spectroscopic measurements [37]. Due to its wide range of applications, FRET has procured ample attention from theoretical and experimental scientists during the recent years. The basic concepts of FRET have now been well developed. However, open questions still exist within the domain. Owing to its limited spread, obtaining an enhanced FRET rate is vital in achieving a wider FRET range. Most of the research focused on this is based on the utilization of the phenomena of surface plasmon resonance of a nearby metallic particle. Although these methods provide enhancement of FRET rate, the control over the maximum achievable FRET rate is limited. In contrast, the method we discuss in this paper significantly enhances the FRET rate in a completely controllable manner by varying the electrical potential on an interacting MNP next to donor-acceptor pair.

It is known that the energy transfer levels identified when there are multiple acceptors is noticeably higher than the FRET efficiency expected from single donoracceptor pairs [38]. There is experimental evidence which suggests that the FRET performance can be enhanced by altering the system configurations [39]. The impact of a third molecule on FRET rate between a donor-acceptor pair has also been studied in [40, 41]. Furthermore, laserassisted resonance energy transfer has been first proposed in [42], which indicates that the properties of FRET can be successfully utilized to develop optical switches. It is shown that the transfer rate can be significantly increased by propagating a laser beam through particles [43]. All these prior studies propose different mechanisms to modify FRET between nanostructures by changing the surrounding objects or the behavior of the nanostructures where FRET occur. However, the third body introduced in such studies is often electrically-neutral and the impact of a MNP on FRET with an external potential applied on it is yet to be studied.

In this paper, we demonstrate that a further enhancement of FRET rate can be achieved by imposing an electrical potential on a neighboring MNP placed between a donor-acceptor pair. Our calculations are based on multiscattering electromagnetic Green's tensor method [44, 45] assuming a spatially persistent local dielectric response for the MNP which is then used to express the FRET rate in the system. When the neighboring particle carry excess electrical potential on it, the induced electric field at the donor position causes the donor dipole moment to change. Although a similar impact is there on the acceptor molecule this does not make an impact on the calculation procedure as the FRET rate is determined

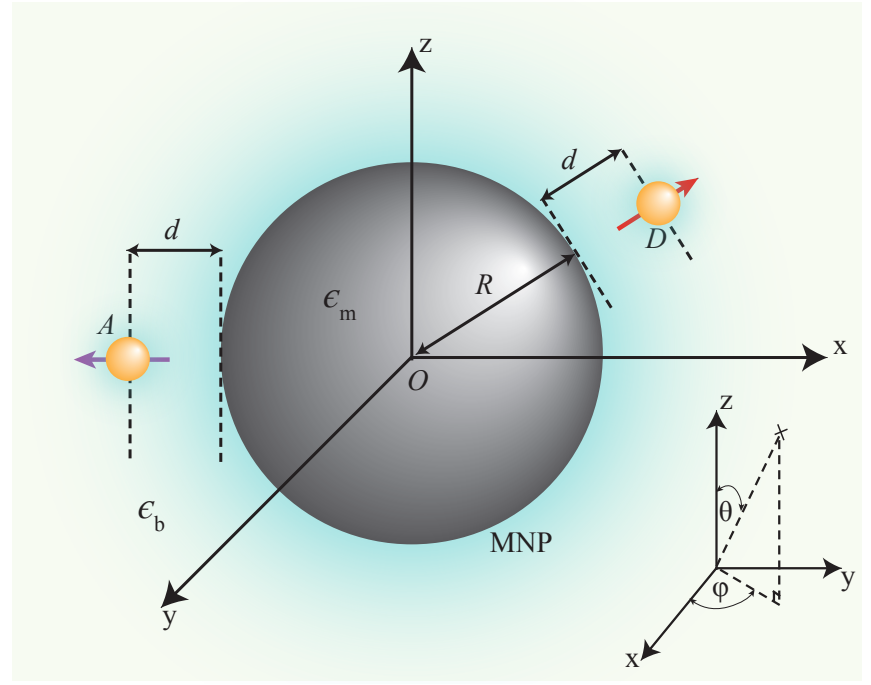

FIG. 1. The Schematic diagram of a MNP and the molecules placed in a water bath of relative permittivity $\epsilon_{\mathrm{b}}=1.77$. The MNP is a Silver $(\mathrm{Ag})$ sphere with radius $R=10 \mathrm{~nm}$ and complex relative permittivity $\epsilon_{\mathrm{m}}$. The centre-to-centre separation between MNP and molecule is $R+d$. Red and purple arrows show the direction of the dipole moments of the excited donor molecule (D) and the ground state acceptor molecule (A) respectively.

by the electric field induced at the acceptor position due to the donor dipole moment. This induced dipole moment of the donor molecule changes the electric field at the acceptor position and the FRET rate between the molecules change due to this. With the potential applied on the MNP, the observed FRET rate enhancement is spread over a wider frequency range compared to the case with a electrically-neutral nanosphere. Furthermore, we study the impact of the relative location of the donor molecule, separation between the MNP and molecules as well as the size of the MNP on FRET rate.

This paper is organized as follows. Following the introduction in this section, Sec. II outlines the formalism including the model and a detailed description of the analytical approach we deploy for our analysis. In Sec. III we discuss the results obtained for certain selected scenarios with a Silver (Ag) MNP and in Sec. IV we summarise and conclude the paper.

\section{FORMALISM}

We place an excited donor molecule (D) and a ground state acceptor molecule (A) near a spherical MNP as shown in Fig. 1. The amount of electrical potential applied on the MNP is taken to be $V_{\text {MNP. }}$. We mainly focus on the influence of this spherical body on the FRET rate between the considered molecules. Initially, we analyze the impact of the position of the $\mathrm{D}$ on FRET rate. Thereafter, for further analysis on the FRET rate, we select the specific donor-acceptor configuration where donor is lo- 
cated at $\theta=\pi / 2, \phi=0$. Here, $\theta$ and $\phi$ refers to the azimuthal and polar angles which are shown in the lower insert of Fig. 1. We analyze the impact of the separation distance between the molecules and the MNP on FRET rate. Furthermore, we study how the various size of the MNP ( i.e. by varying its radius, $R$ ) influence on the FRET rate.

\section{A. Electric field at a point in the presence of a charged spherical MNP}

It is possible to show using the Gauss theorem that a uniform spherical distribution of charges can be replaced by a point charge containing all its charge at the centre of the sphere [46]. Therefore, the total electric field induced by the MNP is determined by this point charge and the electric field induced due to the oscillation of the surface electrons of the MNP. The electric field induced due to the applied potential on the MNP is assumed to be independent of the MNP properties while the field induced by the surface plasmon oscillations are determined by dielectric properties of the MNP.

For an isolated conductor self-capacitance, which is the amount of electric charge that must be added to raise its electrical potential by one unit, is given by the well known equation:

$$
Q=\mathcal{C} \triangle V
$$

The capacitance of a MNP of radius $R$ submerged in a dielectric medium is written as $[47,48]$ :

$$
\mathcal{C}=4 \pi \epsilon_{\mathrm{b}} \epsilon_{0} R+\mathcal{C}_{\text {sur }}
$$

where $\epsilon_{0}$ is the permittivity of vacuum. We ignore the capacitance of the submerged medium $\left(\mathcal{C}_{\text {sur }}=0\right)$, reasoning that it is smaller in orders of magnitude compared to the self capacitance of the particle $[47,48]$. Hence, the capacitance of the MNP is proportional to the radius $R$ and depends on the relative permittivity of the dielectric medium, $\epsilon_{\mathrm{b}}$.

The energy required to add an extra unit charge to the MNP is called charging energy which is given by, $E_{\mathrm{C}}=\frac{e^{2}}{2 \mathcal{C}}$ where $e$ is the charge of an electron [47]. If thermal energy of the electron is not greater than charging energy $E_{\mathrm{C}}$, the electric transport through the nanoparticle is blocked and this is called Coulomb blockade. The blockade can be overcome by applying a sufficiently large bias voltage which is identified as, $\left|V_{\text {bias }}\right|>\frac{e}{2 \mathcal{C}}$. Therefore, all the voltage values considered in this paper falls within this region and the method of applying a voltage to the MNP is explained in [47]. We use Eq. (1) and Eq. (2) to calculate the charge on the MNP by applying a known potential.

The electric field induced due to the MNP at a given point $\vec{r}$ which is located outside the MNP $\left(\left|\vec{r}-\vec{r}_{\mathrm{NP}}\right|>R\right.$.), with $Q$ denoting the charge carried by the MNP can be written as:

$$
\left.\vec{E}\right|_{\vec{r}}=\kappa \frac{Q\left(\vec{r}-\vec{r}_{\mathrm{NP}}\right)}{\left|\vec{r}-\vec{r}_{\mathrm{NP}}\right|^{3}}
$$

where $\kappa=\frac{1}{4 \pi \epsilon_{0}}$ and $\vec{r}_{\mathrm{NP}}$ is the centre of the MNP. However, due to the electrical potential of the MNP, there is an induced dipole moment at the donor molecule. We write this induced dipole moment as:

$$
\vec{\mu}_{\text {Ind }}=\left.\alpha_{\mathrm{D}}(\omega) \vec{E}\right|_{\vec{r}=\vec{r}_{\mathrm{D}}}
$$

where, $\alpha_{\mathrm{D}}(\omega)$ is the polarizability of the donor molecule and $\left.\vec{E}\right|_{\vec{r}=\vec{r}_{\mathrm{D}}}$ is the electric field at the position of the donor due to the charged particle.

\section{B. Classical Green's tensor of the system}

Dyadic Green's function $\bar{G}\left(\overrightarrow{r_{\mathrm{u}}}, \overrightarrow{r_{\mathrm{v}}}, \omega\right)$ is a representation of the electric field at position $\overrightarrow{r_{\mathrm{u}}}$ due to a unit source dipole placed at position $\overrightarrow{r_{\mathrm{v}}}$, with $\omega$ denoting the transition frequency. Since the field at point $\overrightarrow{r_{\mathrm{u}}}$ is decided by the direction of the dipole moment of the source dipole, Green's function must account for all possible orientations as a tensor [44].

This is also called the classical Green's tensor of the system and is the solution of the inhomogeneous Helmholtz equation [49]:

$$
\begin{aligned}
& {\left[\nabla \times \frac{1}{\mu\left(\overrightarrow{r_{\mathrm{u}}}, \omega\right)} \nabla \times \frac{-\omega^{2}}{c^{2}} \epsilon\left(\overrightarrow{r_{\mathrm{u}}}, \omega\right)\right] \bar{G}\left(\overrightarrow{r_{\mathrm{u}}}, \overrightarrow{r_{\mathrm{v}}}, \omega\right)} \\
& =\delta\left(\overrightarrow{r_{\mathrm{u}}}-\overrightarrow{r_{\mathrm{v}}}\right),
\end{aligned}
$$

defined altogether with the boundary condition $\bar{G}\left(\overrightarrow{r_{\mathrm{u}}}, \overrightarrow{r_{\mathrm{v}}}, \omega\right) \rightarrow 0$ for $\left|\overrightarrow{r_{\mathrm{u}}}-\overrightarrow{r_{\mathrm{v}}}\right| \rightarrow \infty$, where, $\epsilon\left(\overrightarrow{r_{\mathrm{u}}}, \omega\right)$ is the dielectric function in position $\overrightarrow{r_{\mathrm{u}}}, \mu\left(\overrightarrow{r_{\mathrm{u}}}, \omega\right)$ is the magnetic permeability of the medium and $c$ is the speed of light in vacuum.

\section{Total energy transfer rate between donor and acceptor}

Total energy transfer rate between two molecules is given by [50]:

$$
\frac{\gamma_{\mathrm{D} \rightarrow \mathrm{A}}}{\gamma_{\mathrm{o}}}=\frac{P_{\mathrm{D} \rightarrow \mathrm{A}}}{P_{\mathrm{o}}}
$$

where $\gamma_{\mathrm{D} \rightarrow \mathrm{A}}$ is the total energy transfer rate from $\mathrm{D}$ to $\mathrm{A}, \gamma_{\mathrm{o}}$ is the decay rate of $\mathrm{D}$ in the absence of $\mathrm{A}, P_{\mathrm{D} \rightarrow \mathrm{A}}$ is the energy absorbed by A per unit time and $P_{\mathrm{o}}$ is the energy emitted by D within a unit time if $\mathrm{A}$ is absent. Starting from Eq. (6) we arrive at [45]:

$$
\begin{aligned}
& \gamma_{D \rightarrow A}=\frac{2\left|\overrightarrow{\mathrm{D}}_{\mathrm{D}}\right|^{2}}{\hbar} \int_{0}^{\infty} f_{\mathrm{D}}(\omega) \alpha_{2}(\omega) v^{2} \\
& \times\left|\vec{n}_{\mathrm{A}} \cdot \bar{G}\left(\vec{r}_{\mathrm{A}}, \vec{r}_{\mathrm{D}} ; \omega\right) \cdot \vec{n}_{\mathrm{D}}\right|^{2} d \omega,
\end{aligned}
$$




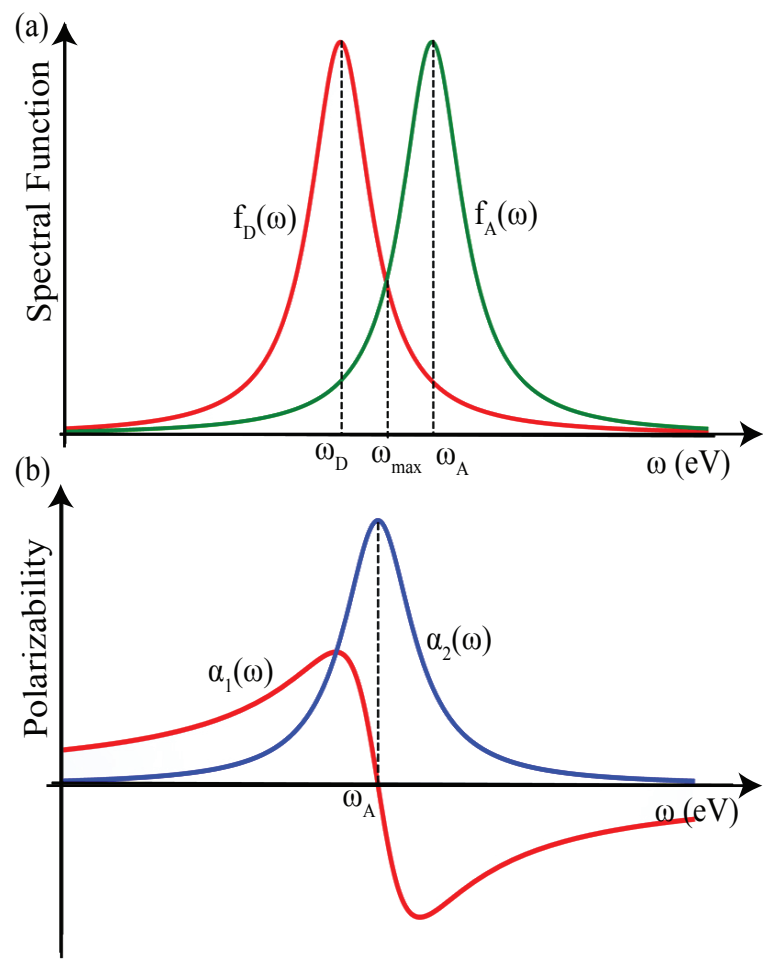

FIG. 2. (a) Spectral overlap between donor emission spectrum $\left(f_{\mathrm{D}}(\omega)\right)$ and acceptor absorption spectrum $\left(f_{\mathrm{A}}(\omega)\right)$. (b) Real $\left(\alpha_{1}(\omega)\right)$ and Imaginary $\left(\alpha_{2}(\omega)\right)$ parts of the polarizability of the acceptor molecule.

as an expression for the total energy transfer rate between two molecules, assuming that the bath is non-magnetic. Here, $\vec{\mu}_{\mathrm{D}}$ is the dipole moment of the donor molecule, $\vec{n}_{\mathrm{A}}$ and $\vec{n}_{\mathrm{D}}$ represent the unit vectors in the directions of the dipole moments, $f_{\mathrm{D}}(\omega)$ is the donor emission spectrum, $\hbar$ is the reduced Planck constant, $\alpha_{2}(\omega)$ is the imaginary part of polarizability of the acceptor given by Eq. (9b), $v=\frac{\omega^{2}}{\epsilon_{\mathrm{o}} c^{2}}$. The complex polarizability is assumed to be isotropic and of the form [51]:

$$
\alpha(\omega)=\alpha_{1}(\omega)+i \alpha_{2}(\omega)
$$

Fig. 2(b) shows its real and imaginary parts expressed as:

$$
\begin{aligned}
& \alpha_{1}(\omega)=\frac{\mu_{\mathrm{A}}^{2}}{\hbar} \frac{\omega_{\mathrm{A}}-\omega}{\left(\omega_{\mathrm{A}}-\omega\right)^{2}+\gamma_{\mathrm{A}}^{2}}, \\
& \alpha_{2}(\omega)=\frac{\mu_{\mathrm{A}}^{2}}{\hbar} \frac{\gamma_{\mathrm{A}}}{\left(\omega_{\mathrm{A}}-\omega\right)^{2}+\gamma_{\mathrm{A}}^{2}},
\end{aligned}
$$

with $\gamma_{\mathrm{A}}$ denoting the half-width of the acceptor absorption spectrum, $\omega_{\mathrm{A}}$ denoting the central frequency of acceptor spectrum and $\mu_{\mathrm{A}}$ denoting the acceptor dipole moment.

\section{Expansion of the dynamic Green's tensor}

Aggregate energy transmitted between the two molecules consist of other energy mechanisms in addition to Förster energy. Since we predominantly focus on FRET, we deduce Eq. (7) to symbolize only the FRET rate. Consequently at near field proximity, it is not required to use the complete Green's function to describe the energy transfer [52]. It is sufficient to use only the static part of the Green's function. This fact is used in the succeeding calculations associated with FRET.

Dynamic Green's tensor is inscribed as the summation of three terms as [45]:

$$
\begin{aligned}
& \bar{G}\left(\overrightarrow{r_{\mathrm{u}}}, \overrightarrow{r_{\mathrm{v}}} ; \omega\right)=\bar{G}_{\mathrm{R}}\left(\overrightarrow{r_{\mathrm{u}}}, \overrightarrow{r_{\mathrm{v}}} ; \omega\right)+\bar{G}_{\mathrm{S}}\left(\overrightarrow{r_{\mathrm{u}}}, \overrightarrow{r_{\mathrm{v}}} ; \omega\right) \\
& +\frac{1}{\epsilon\left(\overrightarrow{r_{\mathrm{u}}}\right)(\omega / c)^{2}} \delta\left(\overrightarrow{r_{\mathrm{u}}}-\overrightarrow{r_{\mathrm{v}}}\right) \bar{I},
\end{aligned}
$$

where, $\overline{G_{\mathrm{R}}}$ is the resonant dipole-dipole interaction term related to radiative energy transfer between molecules, which becomes significant in the far field for homogeneous media. The term $\bar{G}_{\mathrm{S}}$, which relates to the static dipole-dipole interaction, is the nonradiative energy transfer process associated with FRET and is the term we are primarily interested in. The third term depends on the Dirac delta function $\delta\left(\overrightarrow{r_{\mathrm{u}}}-\overrightarrow{r_{\mathrm{v}}}\right)$, which becomes zero elsewhere except at the source dipole position and $\bar{I}$ is the unit tensor. Therefore, we'll be focusing on the term $\bar{G}_{\mathrm{S}}$ for the remaining calculations.

\section{E. Quasi-static approximation of the FRET rate}

The dimensions of the MNP we use here are much smaller than the wavelength of the incident light. Therefore, the phase of the electric field within the volume of the MNP remains unaltered at any given instance. Additionally, all the distances considered here are small enough to disregard retardation effects. Therefore, the use of the quasi-static approximation is sufficient and justified $[53,54]$.

For inhomogeneous environments, the static part of Green's function is derived from its dynamic counterpart with the aid of the quasi-static approximation as:

$$
\bar{G}_{\mathrm{S}}\left(\overrightarrow{r_{\mathrm{u}}}, \overrightarrow{r_{\mathrm{v}}} ; \omega\right)=\lim _{\omega \rightarrow 0} v \bar{G}\left(\overrightarrow{r_{\mathrm{u}}}, \overrightarrow{r_{\mathrm{v}}} ; \omega\right)
$$

when $\overrightarrow{r_{\mathrm{u}}} \neq \overrightarrow{r_{\mathrm{v}}}$

In the quasi-static regime, the total energy transfer can be reduced to the FRET rate and can be written as:

$$
\gamma_{\mathrm{F}}=\frac{2\left|\overrightarrow{\mu_{\mathrm{D}}}\right|^{2}}{\hbar} \int_{0}^{\infty} f_{\mathrm{D}}(\omega) \alpha_{2}(\omega)\left|\vec{n}_{\mathrm{A}} \cdot \bar{G}_{\mathrm{S}}\left(\vec{r}_{\mathrm{A}}, \vec{r}_{\mathrm{D}} ; \omega\right) \cdot \vec{n}_{\mathrm{D}}\right|^{2} d \omega .
$$

For subsequent usage, this equation is written using a substitution as:

$$
\gamma_{\mathrm{F}}=\frac{2\left|\overrightarrow{\mu_{\mathrm{D}}}\right|^{2}}{\hbar} \int_{0}^{\infty} W_{\mathrm{F}} d \omega
$$


where,

$$
W_{\mathrm{F}}=f_{\mathrm{D}}(\omega) \alpha_{2}(\omega)\left|\vec{n}_{\mathrm{A}} \cdot \bar{G}_{\mathrm{S}}\left(\vec{r}_{\mathrm{A}}, \vec{r}_{\mathrm{D}} ; \omega\right) \cdot \vec{n}_{\mathrm{D}}\right|^{2} .
$$

Defining $W_{\text {FO }}$ as the corresponding term in the absence of the MNP, the FRET rate enhancement at a specific frequency can be represented by:

$$
E_{\mathrm{F}}=\frac{W_{\mathrm{F}}}{W_{\mathrm{FO}}}=\frac{\left|\vec{n}_{\mathrm{A}} \cdot \bar{G}_{\mathrm{S}}\left(\vec{r}_{\mathrm{A}}, \vec{r}_{\mathrm{D}} ; \omega\right) \cdot \vec{n}_{\mathrm{D}}\right|^{2}}{\left|\vec{n}_{\mathrm{A}} \cdot \bar{G}_{\mathrm{S}}^{0}\left(\vec{r}_{\mathrm{A}}, \vec{r}_{\mathrm{D}} ; \omega\right) \cdot \vec{n}_{\mathrm{D}}\right|^{2}}
$$

\section{F. The electrical potential at a point in free space}

Let the position of the molecule $\mathrm{v}$, where, $\mathrm{v}=D$ for donor and $\mathrm{v}=A$ for the acceptor, be $\vec{r}_{\mathrm{v}}$ in spherical coordinates. The dipole moment of the molecule is taken to be $\vec{\mu}_{\mathrm{v}}$. The electrical potential at position $\vec{r}$, induced by the dipole moment when the influence of the MNP is neglected is expressed by:

$$
\phi_{\mathrm{v}}^{0}(\vec{r})=\kappa \frac{\vec{\mu}_{\mathrm{v}} \cdot\left(\vec{r}-\vec{r}_{\mathrm{v}}\right)}{\left|\vec{r}-\vec{r}_{\mathrm{v}}\right|^{3}} .
$$

To simplify Eq. (14) we use the identity:

$$
\frac{\vec{r}-\overrightarrow{r_{\mathrm{v}}}}{\left|\vec{r}-\overrightarrow{r_{\mathrm{v}}}\right|^{3}}=\nabla^{\prime} \frac{1}{\left|\vec{r}-\overrightarrow{r_{\mathrm{v}}}\right|}
$$

where, $\nabla^{\prime}$ denotes differentiation with respect to $\overrightarrow{r_{\mathrm{v}}}$. We write the inverse distance between the two vectors as $\frac{1}{\left|\vec{r}-\overrightarrow{r_{\mathrm{v}}}\right|}=\frac{1}{\sqrt{r^{2}+r_{\mathrm{v}}^{2}-2 r r_{\mathrm{v}} \cos \psi}}$, where, $r=|\vec{r}|, r_{\mathrm{v}}=\left|\vec{r}_{\mathrm{v}}\right|$ and $\psi$ is the angle between two vectors. Adhering to the notation, $r_{<}=\min \left\{r, r_{\mathrm{v}}\right\}, r_{>}=\max \left\{r, r_{\mathrm{v}}\right\}$ we write the inverse distance between two vectors as:

$$
\frac{1}{\left|\vec{r}-\overrightarrow{r_{\mathrm{v}}}\right|}=\left(\frac{1}{r_{>}}\right) \sum_{l=0}^{\infty}\left(\frac{r_{<}}{r_{>}}\right)^{l} P_{l}(\cos \gamma)
$$

By applying the addition theorem for spherical harmonics, $P_{l}(\cos \gamma)=\rho \sum_{\mathrm{m}=-1}^{l} Y_{l}^{\mathrm{m}}(\theta, \phi) Y_{l}^{\mathrm{m}}\left(\theta^{\prime}, \phi^{\prime}\right)^{*}$, where $\rho=\frac{4 \pi}{2 l+1}$, Eq. (16) can be written in terms of spherical harmonics:

$$
\frac{1}{\left|\vec{r}-\overrightarrow{r_{\mathrm{v}}}\right|}=\sum_{l \mathrm{~m}} \rho \frac{r_{<}^{l}}{r_{>}^{1+1}} Y_{\theta, \phi}^{l \mathrm{~m}}\left(Y_{\theta^{\prime}, \phi^{\prime}}^{l \mathrm{~lm}}\right)^{*}
$$

Here, we use the notation $Y_{\theta, \phi}^{l \mathrm{~m}}=Y_{l}^{\mathrm{m}}(\theta, \phi)$ and $\sum_{l \mathrm{~m}}=$ $\sum_{l=0}^{\infty} \sum_{m=-1}^{l}$. By using Eq. (15), Eq. (17) and the definition of $\nabla$ in spherical coordinates, we arrive at Eq. (18), which denotes the electrical potential at a point in free space induced by a dipole moment. This is written by taking the center of the coordinate system as origin. We derived Eq. (19), by altering the reference point to the center of the MNP $\vec{r}_{\mathrm{NP}}\left(r_{\mathrm{NP}}, \theta_{\mathrm{NP}}, \phi_{\mathrm{NP}}\right)$ and for the simplification we use $\vec{B}$. Therefore Eq. (19) can be used with a MNP located at any point in the spherical coordinate system.

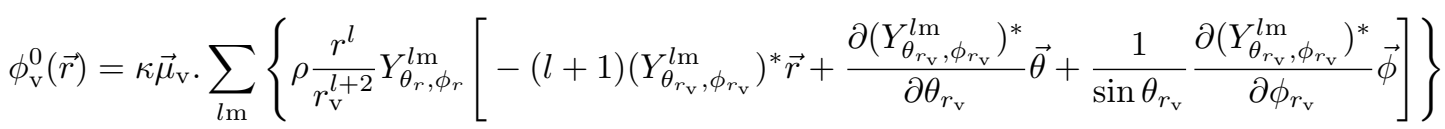

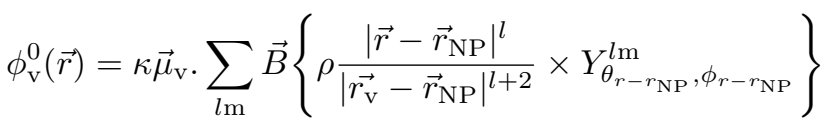

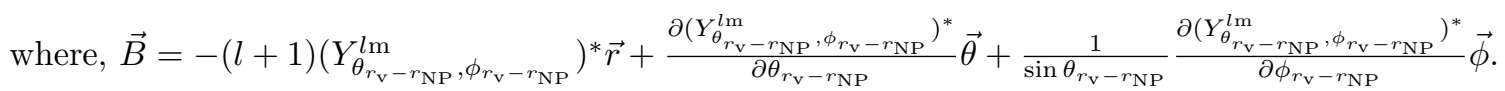

\section{G. electrical potential at a point in the presence of a charge-free spherical MNP}

By solving the Laplace's equation of potential, $\nabla^{2} \phi(\vec{r})=0$ for a charge-free region using the variable separation method, and boundary conditions having azimuthal symmetry, potential can be written as [55]:

$$
\phi(\vec{r})=\sum_{l=0}^{\infty}\left(A_{l} r^{l}+B_{l} r^{-(l+1)}\right) P_{l}(\cos \theta)
$$

The potential inside and outside the MNP can be written as:

$$
\phi(\vec{r})= \begin{cases}\sum_{l=0}^{\infty}\left(A_{l}\left(\frac{r}{R}\right)^{l}+B_{l}\left(\frac{R}{r}\right)^{(l+1)}\right) P_{l}(\cos \theta), & \text { if } r<R . \\ \sum_{l=0}^{\infty}\left(A_{l}^{\prime}\left(\frac{r}{R}\right)^{l}+B_{l}^{\prime}\left(\frac{R}{r}\right)^{(l+1)}\right) P_{l}(\cos \theta), & \text { otherwise. }\end{cases}
$$

In Eq. $21 B_{l} \neq 0$ and $r=0$ results in an nonphysical infinite potential at the center of the MNP. Therefore, $B_{l}=0 \forall l$. Far from the MNP $(r \gg R)$, the influence of the MNP is negligible. Therefore, $\phi(\vec{r})$ is given by Eq. (18). The value of $A_{l}^{\prime}$ can be found using Eq. (18). Afterwards, by applying the boundary conditions for the 


\section{Charged MNP}

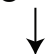

Modified Electric field at the donor position

Modified donor dipole moment

(Initial + Induced dipole moment)

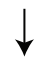

Enhanced electric field at the acceptor position

(Enhancement due to both induced dipole moment of the donor and surface electron oscillation of the MNP)

\section{Enhancement of FRET rate}

FIG. 3. Flow diagram representing the impact of the MNP on the FRET rate of the MNP-molecule system. continuity of the scalar potential:

$$
\left.\phi(\vec{r})\right|_{r=R-0^{+}}=\left.\phi(\vec{r})\right|_{r=R+0^{+}},
$$

and the normal component of the electric displacement vector $\left(D=-\epsilon_{\mathrm{o}} \nabla \phi\right)[56]$ :

$$
-\left.\epsilon_{\mathrm{m}} \frac{\partial \phi(\vec{r})}{\partial r}\right|_{r=R-0^{+}}=-\left.\epsilon_{0} \frac{\partial \phi(\vec{r})}{\partial r}\right|_{r=R+0^{+}},
$$

with Eq. (21) we derive the potential inside and outside the MNP as Eq. (25).

For further simplification, we apply the substitution:

$$
\vec{D}=\frac{l\left(\epsilon_{\mathrm{o}}-\epsilon_{\mathrm{m}}\right)}{\epsilon_{\mathrm{m}} \cdot l+(l+1) \epsilon_{o}} \vec{B} \rho \frac{l R^{2 l+1}}{\left|r_{\mathrm{v}}-r_{\mathrm{u}}\right|^{l+2}}
$$

and write the electrical potential in position $\vec{r}$, induced by the $v^{t h}$ dipole in the presence of the MNP as, Eq. (26).

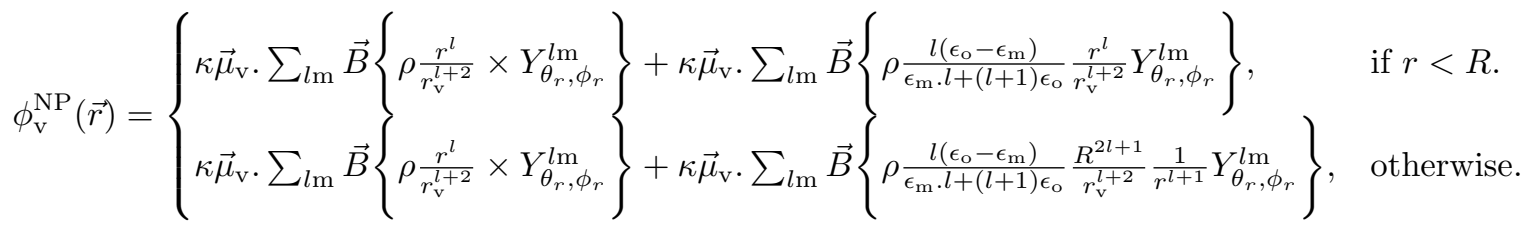

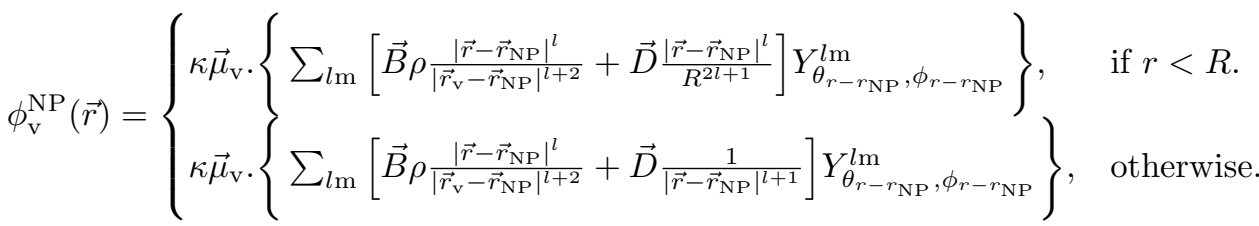

If $l_{\text {Max }}$ is the maximum value of the index $l$ in the spherical harmonic function $Y_{l \mathrm{~m}}$, accuracy of numerical result strongly depends on $l_{\mathrm{Max}}$.

The static Green's tensor is calculated as:

$$
\begin{aligned}
& \vec{n}_{\mathrm{A}} \cdot \bar{G}_{\mathrm{S}}\left(\vec{r}_{\mathrm{A}}, \vec{r}_{\mathrm{D}} ; \omega\right) \cdot \vec{n}_{\mathrm{D}}=-\left.\vec{n}_{\mathrm{A}} \cdot \vec{E}^{(D)}\right|_{\vec{r}=\vec{r}_{\mathrm{A}}} \\
& =\vec{n}_{\mathrm{A}} \cdot\left[\nabla \phi^{(D)}\left(\vec{r}_{\mathrm{A}}\right)\right]
\end{aligned}
$$

where, $\left.\vec{E}^{(D)}\right|_{\vec{r}=\vec{r}_{\mathrm{A}}}$ is the electric field induced by the donor in the acceptor position in the vicinity of the MNP. In the absence of the MNP, the Green's tensor is given by:

$$
\begin{aligned}
& \vec{n}_{\mathrm{A}} \cdot \bar{G}_{\mathrm{S}}^{0}\left(\vec{r}_{\mathrm{A}}, \vec{r}_{\mathrm{D}} ; \omega\right) \cdot \vec{n}_{\mathrm{D}}=-\left.\vec{n}_{\mathrm{A}} \cdot \vec{E}^{0}\right|_{\vec{r}=\vec{r}_{\mathrm{A}}} \\
& =\vec{n}_{\mathrm{A}} \cdot\left[\nabla \phi_{\mathrm{D}}^{0}\left(\vec{r}_{\mathrm{A}}\right)\right] .
\end{aligned}
$$

By incorporating Eq. (1)-(4) with Eq. (26) and substituting in Eq. (13), we obtain the main analytical result of this work.

\section{RESULTS AND DISCUSSION}

We use normalized Lorentzian functions as donoremission and acceptor-absorption spectral functions [57] as shown in Fig. 2(a). To observe FRET, the acceptor molecule must be able to absorb energy from the donor molecule, which means the emission spectrum of donor $\left(f_{\mathrm{D}}(\omega)\right)$ and the absorption spectrum of acceptor $\left(f_{\mathrm{A}}(\omega)\right)$ must overlap. To obtain the aforementioned spectral overlap, we select the frequency of maximum overlap $\left(\omega_{\max }\right)$ to be $3.0 \mathrm{eV}$. Central frequency of donor spectrum, $\omega_{\mathrm{D}}=\omega_{\max }-\zeta$ and the central frequency of acceptor spectrum, $\omega_{\mathrm{A}}=\omega_{\max }+\zeta$ where,$\zeta=0.1 \mathrm{eV}$ and $\Gamma=0.08 \mathrm{eV}$ which is the half-width of the Lorentzian shape .

We have used experimental data in the reference [58] for determining the dielectric permittivity of $\mathrm{Ag}$, where the background was assumed to have a spatially nonvarying dielectric constant. We selected the radius of the metal nanoparticle $(10 \mathrm{~nm})$ to be less than the molec- 
(a)

(d)
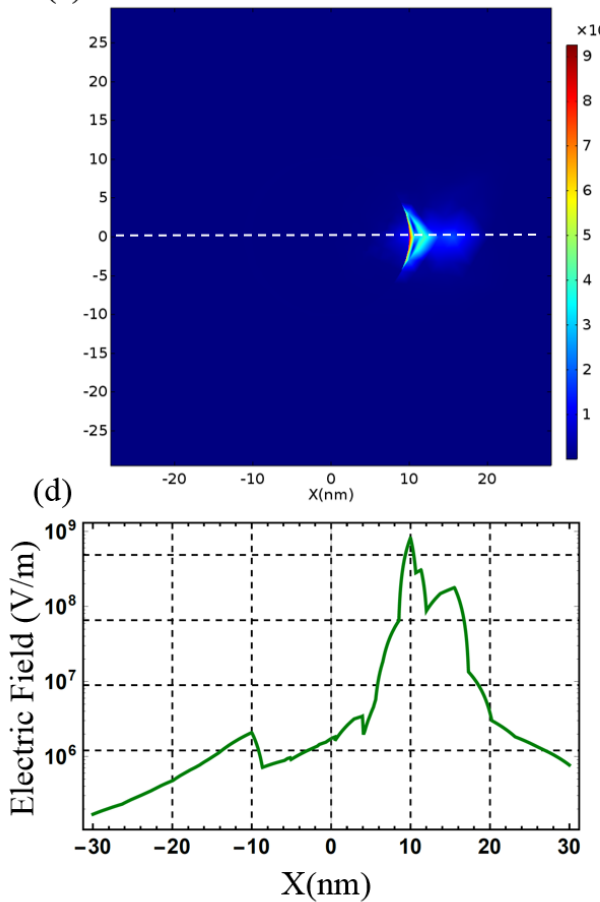

(b)

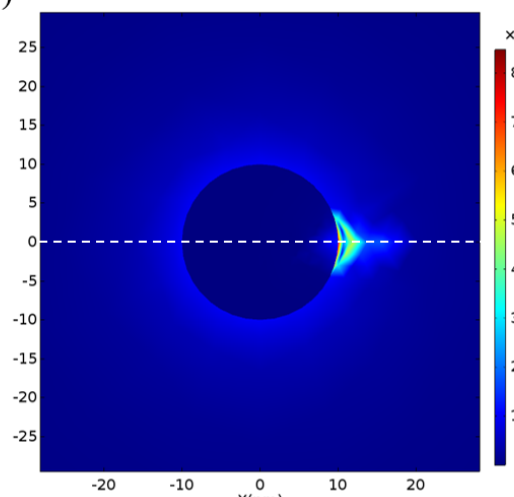

(e)

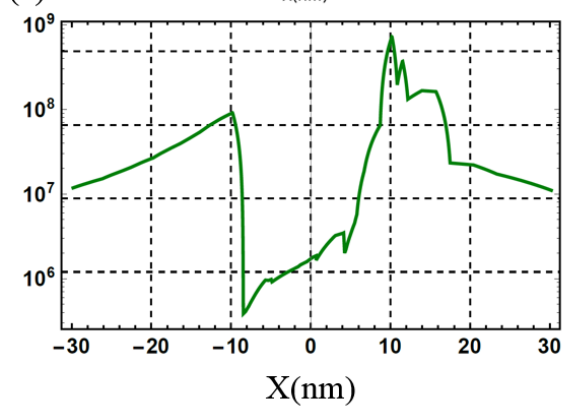

(c)

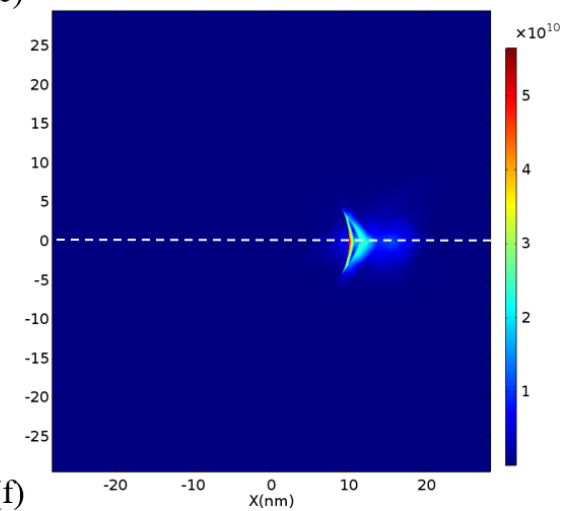

(f)

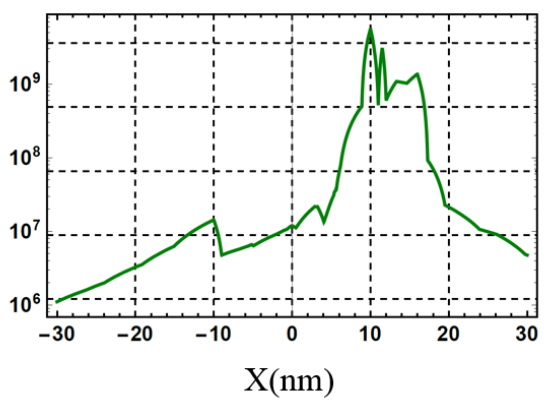

FIG. 4. Images exhibit field patterns of an excited donor molecule with electric dipole moment $1.602176 \times 10^{-28} \mathrm{Cm}$ in the absence of acceptor. (a)-(c) represent the electric field distributions while (d)-(f) represent the variation of electric field magnitude along the $\mathrm{x}$ axis shown by white dashed line in each image (a)-(c). Here, $d=2 \mathrm{~nm}, \omega=2.8 \mathrm{eV}$ and $R=10 \mathrm{~nm}$. (a) and (d) correspond to the case where the donor molecule is placed near a Ag MNP with $V_{\text {MNP }}=0 \mathrm{~V}$ while (b) and (e) correlate with a MNP carrying a potential of $V_{\mathrm{MNP}}=1 \mathrm{~V}$. (c) and (f) represent the case where the impact of the potential of the MNP on the donor molecule has been taken into account and the field due to the donor dipole moment has been illustrated here.

\begin{tabular}{lc}
\hline \hline Scenario & $\left.\vec{E}^{(D)}\right|_{\vec{r}=\vec{r}_{\mathrm{A}}}$ \\
\hline (1) Donor in the absence of MNP (No Ag MNP present) & $0.145 \mathrm{MV} / \mathrm{m}$ \\
(2) Donor in the presence of MNP with $V_{\mathrm{MNP}}=0 \mathrm{~V}$ & $1.97 \mathrm{MV} / \mathrm{m}$ \\
(3) Donor in the presence of MNP with $V_{\mathrm{MNP}}=1 \mathrm{~V}$ (By ignoring the impact of MNP charge on Donor molecule) & $86.6 \mathrm{MV} / \mathrm{m}$ \\
(4) Electric field due to donor with the consideration of induced dipole moment & $76.166 \mathrm{MV} / \mathrm{m}$ \\
\hline \hline
\end{tabular}

TABLE I. Electric field at the acceptor position for different scenarios

ular transition wavelength and the separation between the MNP and the molecule to be a few nanometers $(0.5 \mathrm{~nm}-2.0 \mathrm{~nm})$, so that FRET dominates over other energy transfer mechanisms.

The FRET rate depends on the electric field or electrical potential at the acceptor position due to the electric dipole moment of the donor molecule. Fig. 3 represents how the charged MNP influence on changing the electric field at the acceptor position and finally on increasing the FRET rate of the system. The results we discuss here are contingent on the spectral overlap, orientation and the magnitude of the dipole moment which are the key parameters determining FRET rate [59]. Here, we have assumed that the electric field due to the potential of the MNP results only on the change of the electric dipole moment of the donor molecule. Although, the electric field induced at the acceptor position change the acceptor dipole moment, this does not make any impact on the calculation procedure as the FRET rate is determined by the classical Green's tensor. As we observed from Eq. 12 FRET rate is determined by the term $\bar{G}_{\mathrm{S}}\left(\vec{r}_{\mathrm{A}}, \vec{r}_{\mathrm{D}} ; \omega\right)$, which is the electric field at acceptor position due to the source dipole, which is taken to be the donor dipole.

Since the classical Green's function, which is a representation of the electric field is used to calculate the FRET rate, observing potential and static electric field patterns of the system gives an overview of how the FRET rate is influenced by the system. In Fig. 4, we have illustrated the electric field distribution of the system for different scenarios specified. Electric field values 
we obtained at the acceptor position for these scenarios are shown in Table. I.

From Table. I we can observe that for all scenarios considered, field at the acceptor is larger compared to when the MNP is absent (comparison of case (1) in table with (2),(3) and (4) which are illustrated by Fig. 4). The FRET rate near the Ag particle is larger than that of unbound donor-acceptor pairs $[57,60]$ due to the plasmon resonances originating from surface plasmon excitations. This is substantiated by the results we obtained. The electric field magnitude at the acceptor in the presence of the MNP is $1.97 \mathrm{MV} / \mathrm{m}$ as shown in the table while this is only $0.145 \mathrm{MV} / \mathrm{m}$ in the absence of the MNP. These results are in agreement with previous research $[38,39]$. What we have shown here is that having a charged MNP will further increase this value. For a $1 \mathrm{~V}$ potential applied on the MNP, the observed electric field at the acceptor position due to the donor dipole moment is $76.166 \mathrm{MV} / \mathrm{m}$. This is significantly large (38 times) compared to when there is no excess electrical potential applied on the MNP. Scenario (3)(Fig. 4 (b) and (e)) represents the total electric field at the acceptor position due to both donor dipole moment and the charge on the MNP. However, the FRET rate depends only on the electric field due to dipole moment of the donor molecule. Therefore, in scenario (4), which correspond to Fig. 4 (c) and (f), we have ignored the field induced due to the electrical potential of the MNP while we have contemplated the impact of the induced dipole moment. From these observed electric field values we can conclude that the applied electrical potential on the MNP makes an significant impact on the FRET rate between the molecules and in this paper we'll be studying this impact.

\section{A. Impact of the position of the donor molecule on FRET rate}

In this section we analyze the impact of the relative position of the donor molecule on the observed FRET rate of the system. As observed in Fig. 5(a) and Fig. 5(b) we notice that the FRET rate depends on the location of the donor. When the location of the donor is changed by changing $\theta$ and $\phi$ FRET rate varies in a larger span as observed from the figure. Furthermore, by comparing Fig. 5(a) and Fig. 5(b), when $1 \mathrm{~V}$ potential is applied on the MNP, observed values of FRET rate enhancement is significantly larger compared to the charge-free MNP case. But, the pattern of variation is much similar in both cases. For both cases maximum FRET rate enhancement correspond to the same range of values of $\theta$ and $\phi$. For the considered frequency and separation distances the FRET rate enhancement observed for the charged MNP for each $\theta, \phi$ pair is more than 100 times larger compared to the charge-free MNP case. For all the other simulations we consider in this paper, $\theta$ and $\phi$ are kept fixed as $\theta=\pi / 2$ and $\phi=0$. (a)

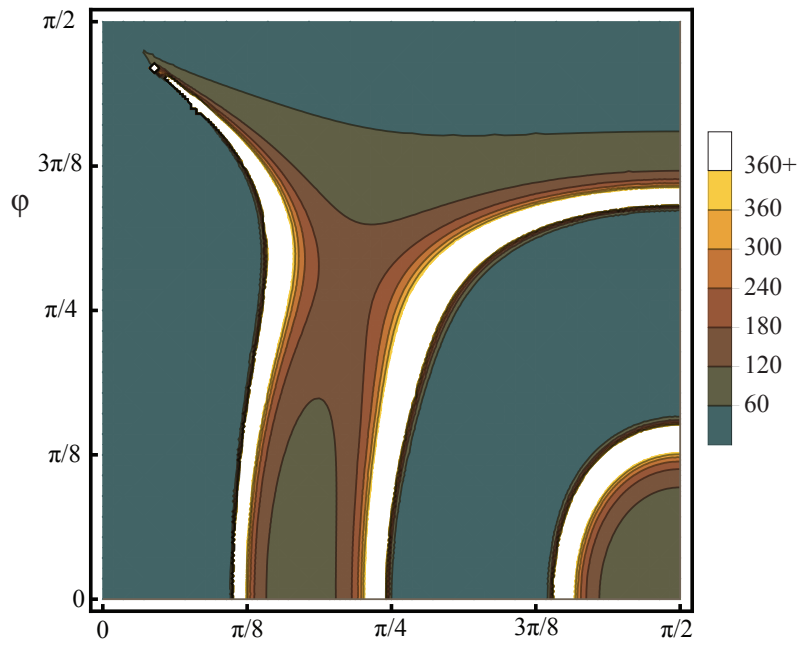

(b)

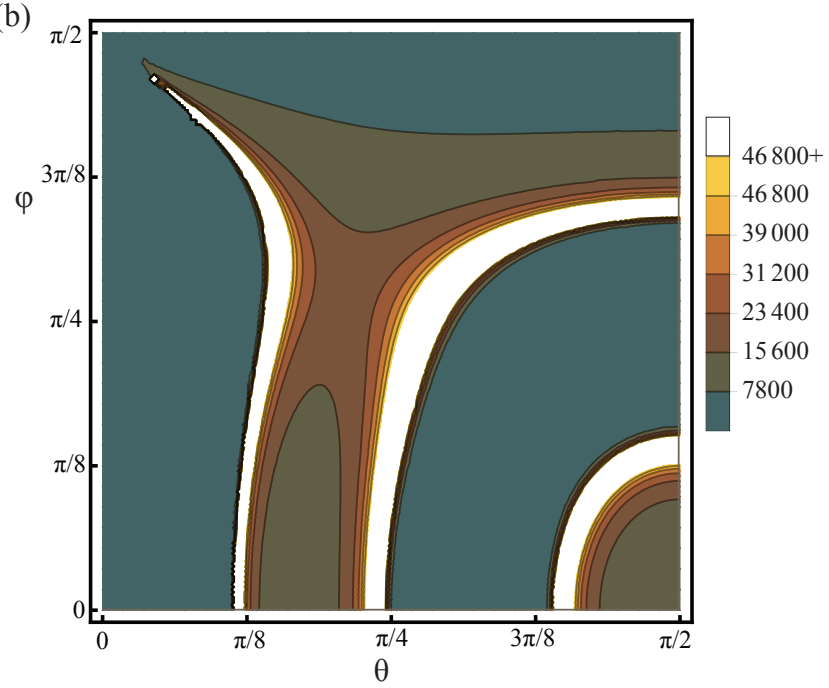

FIG. 5. Enhancement of FRET rate between the molecules positioned in the presence of an Ag MNP for different positions of the donor molecule. (a) Donor-Acceptor in the presence of charge-free MNP. (b) Donor-Acceptor in the presence of charged MNP with a potential of $V_{\mathrm{MNP}}=1 \mathrm{~V}$ applied on the MNP. $d=0.5 \mathrm{~nm}$ and $\omega=2.8 \mathrm{eV}$ is kept fixed for both cases. $\theta$ and $\phi$ correspond to the azimuthal angle and polar angle respectively as shown in Fig. 1.

\section{B. Impact of amount of potential applied on the MNP on FRET rate}

Fig. 6 depicts the resemblance of the FRET rate enhancement in the presence of a charged and charge-free spherical MNP as a function of the transition frequency for different values of potential applied on the MNP $\left(V_{\mathrm{MNP}}\right)$ for a pair of molecules. Electric dipole moments are aligned with the axis of symmetry of the system. We apply different potentials on the MNP and obtain simulation results for FRET rate enhancement at a given frequency, as given by Eq. (13). 

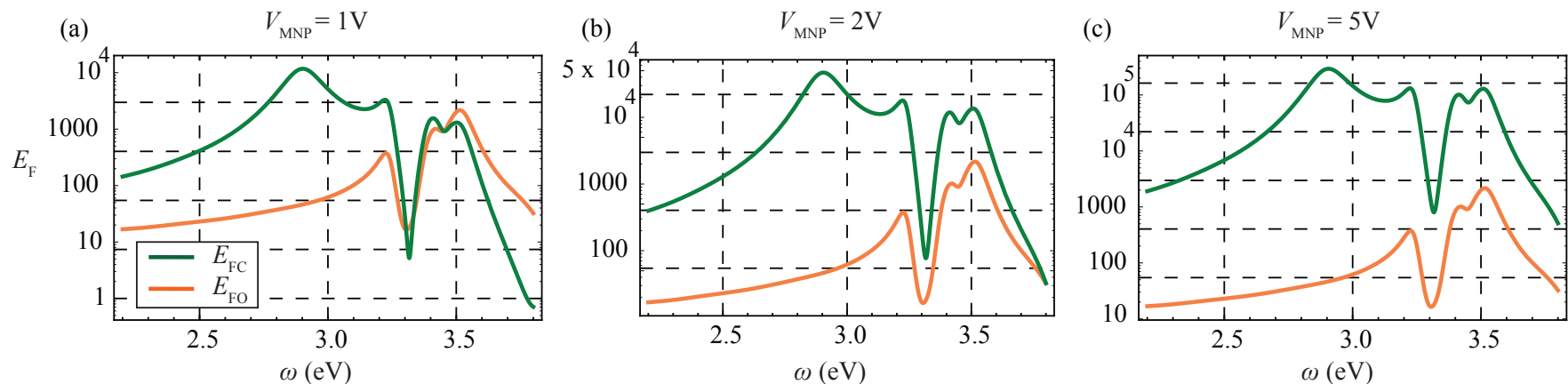

FIG. 6. Enhancement of FRET rate between two molecules positioned in the presence of an Ag MNP for different potentials applied on the MNP. Geometry and coordinates of the corresponding system are similar to Fig. 1 where $\theta=\pi / 2, \phi=0$. The green $\left(E_{\mathrm{FC}}\right)$ and orange $\left(E_{\mathrm{FO}}\right)$ line correspond to enhancement of FRET rate for a charged MNP and enhancement of FRET for a charge-free MNP. $V_{\text {MNP }}$ is the potential applied on the MNP. Results corresponding to $V_{\mathrm{MNP}}=1 \mathrm{~V}, 2 \mathrm{~V}$ and $5 \mathrm{~V}$ are shown by (a), (b) and (c) respectively.

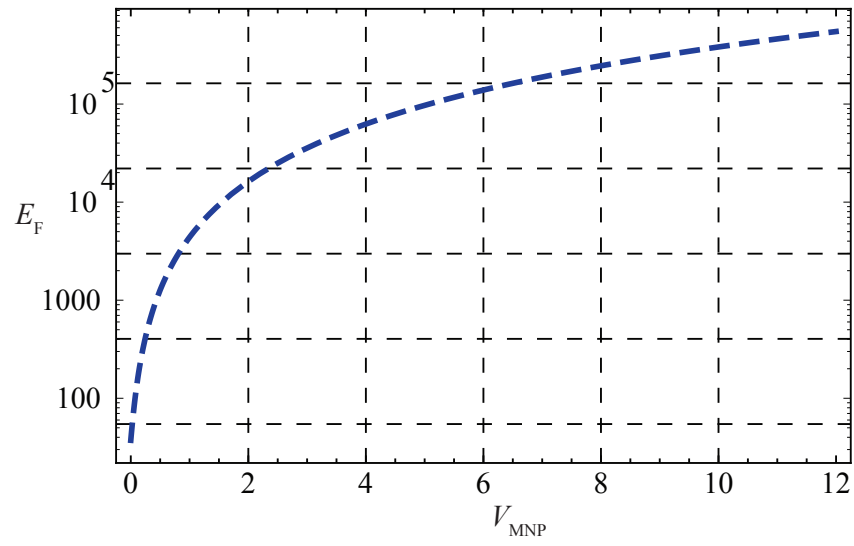

FIG. 7. FRET rate enhancement $\left(E_{\mathrm{F}}\right)$ versus voltage applied on the MNP.

The green and orange lines in Fig. 6(a)-(c) correspond to $E_{\mathrm{FC}}$ and $E_{\mathrm{FO}}$, respectively where, $E_{\mathrm{FC}}$ is the enhancement of FRET rate for a charged MNP case, while $E_{\mathrm{FO}}$ is for the case containing zero excess charge on the MNP. We emphasize that there is a significant enhancement of the FRET rate depending on the amount of potential applied on the Ag MNP as the gap between the two curves for a specified frequency increases with the potential. As shown in Fig. 6, for an applied potential of $1 \mathrm{~V}$, the maximum enhancement achieved is nearly $10^{4}$, while it is more than $10^{5}$ for $5 \mathrm{~V}$, which is approximately 10 times larger. For FRET based applications, this will be a very important phenomenon.

We notice that the FRET rate enhancement highly depend on frequency. For the charge-free case, results obtained tally with previous observations [52]. Enhancement values we obtain differ due to the selection of maximum spectral overlap frequency and dipole moments. For the Ag MNP we consider, outside the considered frequency range, the FRET rate enhancement diminishes noticeably. Selected frequency range includes the reso- nance range for $\mathrm{Ag}$ [58]. If the donor and acceptor configuration is changed, the observed FRET rate enhancement will differ depending on the orientation of donoracceptor dipole moments, distance from the MNP and the distance between the molecules. However, an impact from the electrical potential on the MNP to the FRET rate would still remain.

Frequency range where we obtain the enhancement of

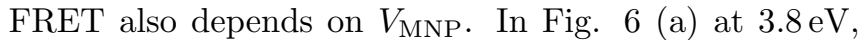
$E_{\mathrm{FC}}<1$ which does not indicate an enhancement but a reduction. However, Fig. $6(\mathrm{~b})-(\mathrm{c})$ provide a $E_{\mathrm{FC}}>1$ for $3.8 \mathrm{eV}$ and the value of $E_{\mathrm{FC}}$ at this frequency is also increasing indicating that effective frequency range where we can obtain an enhancement of FRET is also increasing with $V_{\text {MNP. }}$

The shape of the curve denoting $E_{\mathrm{FC}}$ is largely determined by the polarizability of the donor molecule, emission spectrum of the donor and the selection of maximum spectral overlap frequency. Peaks in the graph correspond to single scattering resonance frequency, determined by the MNP properties.

Fig. 7 shows how $E_{\mathrm{F}}$ changes with the potential applied on the MNP. This result corresponds to the case $d=0.5 \mathrm{~nm}$ and $\omega=2.8 \mathrm{eV}$. This is a further explanation for the results shown in Fig. 6. Although this corresponds to a specific frequency and a separation distance, we will generally observe a similar behavior for other frequencies and separation distances under consideration. According to Fig. 7, we can clearly perceive the enhancement of $E_{\mathrm{F}}$ with increasing $V_{\mathrm{MNP}}$. Furthermore, if we analyze how the static Green's tensor of the system changes with $V_{\mathrm{MNP}}$, we observe a similar trend as we see in Fig. 7.

\section{Impact of distance dependance on FRET rate}

Fig. 8 provides a comparison of the FRET rate enhancement in the presence of a charged and charge-free spherical MNP as a function of transition frequency for 

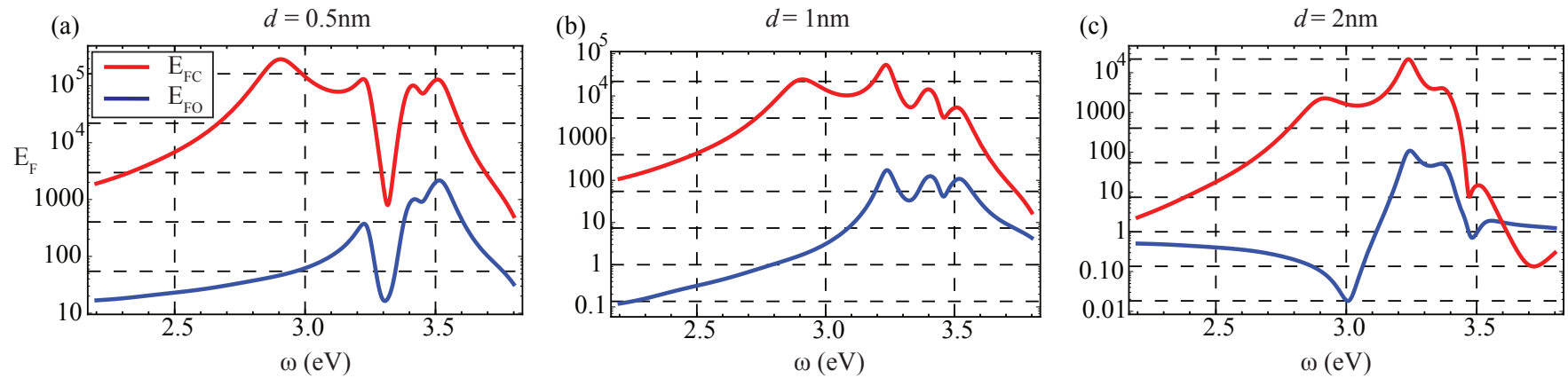

FIG. 8. Enhancement of FRET rate between two molecules positioned in the presence of an $\mathrm{Ag}$ MNP with $V_{\mathrm{MNP}}=5 \mathrm{~V}$ for different separation distances (d). Geometry and coordinates of the corresponding system are similar to Fig.1 where $\theta=\pi / 2, \phi=0$. The red and blue line correspond to enhancement of FRET rate for the charged MNP and a charge-free MNP. Results corresponding to $d=0.5 \mathrm{~nm}, 1 \mathrm{~nm}$ and $2.0 \mathrm{~nm}$ are shown by (a), (b) and (c) respectively.
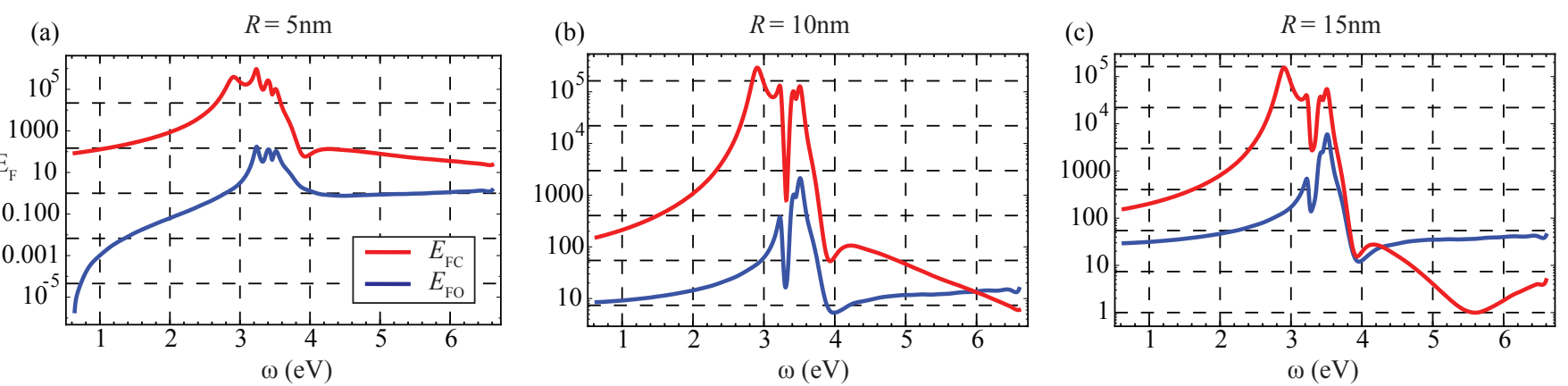

FIG. 9. Enhancement of FRET rate between two molecules positioned in the presence of a $\mathrm{Ag}$ MNP for $d=0.5 \mathrm{~nm}$ for various sizes of spheres. The red and blue line correspond to the charged MNP containing $5 \mathrm{~V}$ potential and $0 \mathrm{~V}$ potential, respectively. Results corresponding to $R=5 \mathrm{~nm}, 10 \mathrm{~nm}$ and $15 \mathrm{~nm}$ are shown by (a), (b) and (c) respectively.

different $d$. We apply a potential of $5 \mathrm{~V}$ on the MNP and obtain simulation results for the enhancement of FRET rate $E_{\mathrm{F}}$ at a given frequency. Once $d$ changes, relative separation between donor and acceptor changes.

We can observe that the maximum enhancement of FRET $\left(E_{\mathrm{F}}\right)$ decreases with the increasing separation distance. This is valid for both charged and charge-free MNP cases. For, $d=0.5 \mathrm{~nm}$, the observed maximum enhancement is more than $10^{5}$ at $2.9 \mathrm{eV}$, while it has been reduced to $10^{4}$ at $3.2 \mathrm{eV}$ for $2.0 \mathrm{~nm}$ distance for the charged particle scenario. However, this maximum values are nearly $10^{3}$ and $10^{2}$ if the particle is charge-free. Therefore, we observe that the maximum $E_{\mathrm{F}}$ increases by nearly $10^{2}$ for all the distances for a given frequency. For all selected separation distances in general, we observe that $E_{\mathrm{FO}}<E_{\mathrm{FC}}$. However, the span of the frequency range we observe an enhancement, reduces with increasing separation distance.

\section{Impact of nanoparticle size on FRET rate}

Results discussed so far, focus only on the case with $R=10 \mathrm{~nm}$. However, for a fixed separation distance the FRET rate enhancement as well as the frequency range, where we observe such an enhancement strongly depends on the MNP radius as evident from Fig. 9. As observed in Fig. 9(a), for the entire frequency range we considered, $E_{\mathrm{FC}}>E_{\mathrm{FO}}$. However, frequency range where $E_{\mathrm{FC}}>E_{\mathrm{FO}}$ reduces with increasing MNP size as observed in Fig. 9(a)-(c). Furthermore, the maximum obtained enhancement also shows a slight reduction with the increasing MNP size when the MNP is charged. This is due to the increasing relative separation between the donor-acceptor molecules. With increasing MNP size the induced dipole moment at the donor molecule reduces as the electric field induced at the donor due to the MNP is reduced. Therefore, the electric field at the acceptor due to donor dipole moment is also reduced resulting the observed reduction of the FRET rate enhancement $E_{\mathrm{FC}}$ in Fig. 9.

\section{SUMMARY AND CONCLUSION}

FRET plays a paramount role in a range of applications, as it becomes noticeable when the intermolecular separation between the donor-acceptor particles is a few nanometers. However, due to its higher order inverse distance dependence, it gets drastically weaker with in- 
creasing distance which is unpropitious for applications, which rely on it. Therefore, increasing the FRET range is commendatory and has been extensively studied in the literature. In this work, we have presented an approach to achieve enhancement in the FRET rate over a broad spectrum by placing a spherical MNP with an electrical potential applied on it, in the vicinity of the energy exchanging molecules.

Our calculations based on the exact multiscattering electromagnetic Green's function approach indicate that the amount of electrical potential applied on the MNP has a direct influence on the FRET rate. The FRET rate enhancement increases remarkably with the potential applied on the MNP. Furthermore, we analyzed the impact of separation between the molecules and the MNP on the FRET rate. With decreasing separation distance between the molecules and the MNP, the FRET rate enhancement intensifies and we observed a simultaneous increase in the correlating frequency range. Furthermore, we investigated the impact of the MNP radius on FRET rate enhancement, where we perceived a higher FRET rate over a wider frequency range, for larger electricallyneutral MNP radii. However, when an electrical poten- tial is applied on the MNP the maximum FRET rate enhancement shows a slight reduction with increasing MNP size in the considered frequency range as a result of the curtailment of the induced electric field at the acceptor position due to the reduction of the induced dipole moment at the donor molecule. Therefore, the impact of the electrical potential applied on the MNP is significant for smaller MNP radii when the separation distances are fixed.

In essence, applying an electrical potential on a vicinal MNP provides an outstanding further enhancement of FRET over a wider frequency range, in comparison to the previous work done based on the conventional plasmonic resonant field based practices.

\section{ACKNOWLEDGMENTS}

Champi Abeywickrama would like to thank the members of $\mathrm{A} \chi \mathrm{L}$ at Monash University for their encouragement and support. The work of Champi Abeywickrama is supported by the Monash University Institute of Graduate Research.
[1] P. Wu and L. Brand, Analytical Biochemistry 218, 1 (1994).

[2] P. S. Andrea Montali, Cees Bastiaansen and C. Weder, 392, 261 (1998).

[3] G. D. Scholes, Annual Review of Physical Chemistry 54, 57 (2003).

[4] J. S. Avery, Proceedings of the Physical Society 88, 1 (1966).

[5] M. S. Eggleston, K. Messer, L. Zhang, E. Yablonovitch, and M. C. Wu, Proceedings of the National Academy of Sciences 112, 1704 (2015).

[6] D. D.L., jcp 21, 836 (1953).

[7] T. Forster, Naturwissenschaften 33, 166 (1946).

[8] B. W. van der Meer, "Fret - förster resonance energy transfer," (Wiley-VCH Verlag GmbH Co. KGaA, 2013) pp. 23-62.

[9] E. M. Purcell, H. C. Torrey, and R. V. Pound, Phys. Rev. 69, 37 (1946).

[10] D. L. Andrews and D. S. Bradshaw, European Journal of Physics 25, 845 (2004).

[11] D. L. Andrews and D. S. Bradshaw, Annalen der Physik 1, 173-186 (2014).

[12] C. Road, 42, 4291 (1990).

[13] D. L. Andrews and J. M. Leeder, Journal of Chemical Physics 130 (2009).

[14] C. Y. Wong, C. Curutchet, S. Tretiak, and G. D. Scholes, Journal of Chemical Physics 130 (2009).

[15] M. Mohseni, P. Rebentrost, S. Lloyd, and A. AspuruGuzik, The Journal of Chemical Physics 129, 174106 (2008).

[16] E. Rabinowitch, The Journal of Physical Chemistry 61, 870 (1957).

[17] R. van Grondelle, J. P. Dekker, T. Gillbro, and V. Sundstrom, Biochimica et Biophysica Acta (BBA) - Bioener- getics 1187, 1 (1994).

[18] S. Buhbut, S. Itzhakov, E. Tauber, M. Shalom, I. Hod, T. Geiger, Y. Garini, D. Oron, and A. Zaban, ACS Nano 4, 1293 (2010).

[19] S. Chanyawadee, R. T. Harley, M. Henini, D. V. Talapin, and P. G. Lagoudakis, Phys. Rev. Lett. 102, 077402 (2009).

[20] K. Shankar, X. Feng, and C. A. Grimes, ACS Nano 3, 788 (2009).

[21] R. Hildner, D. Brinks, J. B. Nieder, R. J. Cogdell, and N. F. van Hulst, Science 340, 1448 (2013).

[22] D. Andrews, in Tutorials in Complex Photonic Media, edited by M. Noginov, M. McCall, G. Dewar, and N. Zheludev (SPIE Press, Bellingham, WA, 2009) pp. 439-478.

[23] I. L. Medintz, A. R. Clapp, H. Mattoussi, E. R. Goldman, B. Fisher, and J. M. Mauro, Nature Materials 2 (2003).

[24] M. C. Morris, Biochimica et Biophysica Acta (BBA)Proteins and Proteomics , 1387-1395 (2013).

[25] S. Lu and Y. Wang, Clinical Cancer Research 16, 3822 (2010).

[26] G. D. Scholes, , 57 (2003).

[27] R. van Grondelle and V. I. Novoderezhkin, Nature 463, 107 (2010).

[28] S. S. Vogel, C. Thaler, and S. V. Koushik, Science Signaling (2006).

[29] O. T. Hoggan, Philip E., Advances in Quantum Chemistry - Electron Correlation in Molecules - Ab Initio beyond Gaussian Quantum Chemistry, Vol. 73 (Elsevier, 2016).

[30] C. Rupasinghe, I. D. Rukhlenko, and M. Premaratne, ACS Nano 8, 2431 (2014), pMID: 24559464.

[31] C. Jayasekara, M. Premaratne, M. Stockman, and S. D. Gunapala, Journal of Applied Physics, 118 (2015). 
[32] C. Jayasekara, M. Premaratne, S. D Gunapala, and M. Stockman, Journal of Applied Physics, 119, 133101 (2016).

[33] L. Kumarapperuma, M. Premaratne, P. K. Jha, M. Stockman, and G. Agrawal, Applied Physics Letters, 112, 201108 (2018).

[34] T. Warnakula, M. I. Stockman, and M. Premaratne, J. Opt. Soc. Am. B 35, 1397 (2018).

[35] S. Zadran, S. Standley, K. Wong, E. Otiniano, A. Amighi, and M. Baudry, Applied microbiology and biotechnology 96, 895 (2012).

[36] L. Stryer and R. P. Haugland, Proceedings of the National Academy of Sciences 58, 719 (1967).

[37] L. Stryer, Annual Review of Biochemistry 47, 819 (1978).

[38] S. V. Koushik, P. S. Blank, and S. S. Vogel, PLoS ONE 4 (2009).

[39] J. Zhang, Y. Fu, and J. R. Lakowicz, The Journal of Physical Chemistry C 111, 50 (2007).

[40] D. Craig and T. Thirunamachandran, Chemical Physics 135, 37 (1989).

[41] A. Salam, The Journal of Chemical Physics 136, 014509 (2012).

[42] D. L. Andrews and J. M. Leeder, The Journal of Chemical Physics 130, 034504 (2009).

[43] D. Weeraddana, M. Premaratne, S. D. Gunapala, and D. L. Andrews, Physical Review B 94 (2016).

[44] L. Novotny and B. Hecht, Principles of Nano-Optics, 2nd ed. (Cambridge University Press, 2012).

[45] J. A. Gonzaga Galeana and J. R. Zurita-Sanchez, The Journal of Chemical Physics 139, 244302 (2013).

[46] M. Sadiku, Elements of Electromagnetics, Oxford series in electrical and computer engineering (Oxford University Press, 2015).

[47] K. Szaciłowski, Infochemistry: Information Processing at the Nanoscale (John Wiley and Sons Ltd, 2012) Chap. 4.

[48] H. Lehmann, S. Willing, S. Möller, M. Volkmann, and C. Klinke, Nanoscale, 8, 14384 (2016).

[49] S. Y. Buhmann, 248, 287 (2012).

[50] L. Novotny and B. Hecht, Princples of Nano-Optics, Vol. 1 (Cambridge University Press, 2006) Chap. 8.

[51] A. E. Cohen and S. Mukamel, The Journal of Physical Chemistry A 107, 3633 (2003).

[52] J. Ren, T. Wu, B. Yang, and X. Zhang, Phys. Rev. B 94, 125416 (2016).

[53] H. Hapuarachchi, M. Premaratne, Q. Bao, W. Cheng, S. D. Gunapala, and G. P. Agrawal, Physical Review B, 95 (2017).

[54] H. Hapuarachchi, S. Mallawaarachchi, H. T Hattori, W. Zhu, and M. Premaratne, Journal of Physics: Condensed Matter, 30 (2017).

[55] R. D. Artuso, "The optical response of strongly coupled quantum dot-metal nanoparticle hybrid systems," (2012).

[56] G. Mie, Annalen der Physik 330, 377 (1908).

[57] V. N. Pustovit and T. V. Shahbazyan, Physical Review B 83, 085427 (2011).

[58] P. B. Johnson and R. W. Christy, Phys. Rev. B 6, 4370 (1972).

[59] van der Meer B. Wieb, van der Meer Daniel M., and V. S. S., FRET - Förster Resonance Energy Transfer (Wiley-Blackwell, 2013) Chap. 3.

[60] H. Y. Xie, H. Y. Chung, P. T. Leung, and D. P. Tsai, Phys. Rev. B 80, 155448 (2009). 